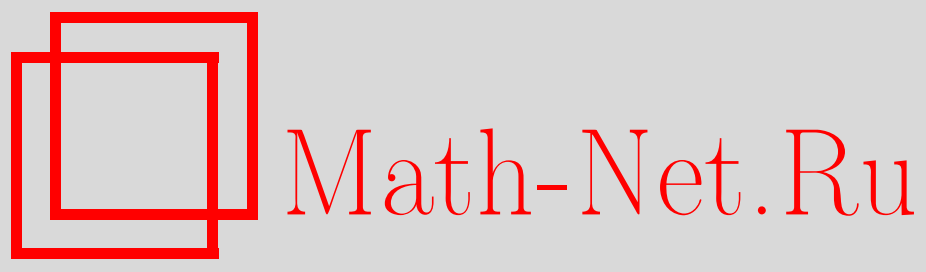

Е. В. Шульман, О некоторых функциональных уравнениях и представлениях топологических полугрупп, Функи. анализ и его прил., 2005, том 39, выпуск $4,83-85$

DOI: https://doi.org/10.4213/faa89

Использование Общероссийского математического портала Math-Net.Ru подразумевает, что вы прочитали и согласны с пользовательским соглашением http://www. mathnet.ru/rus/agreement

Параметры загрузки:

IP : 34.229 .45 .116

26 апреля 2023 г., 13:45:24

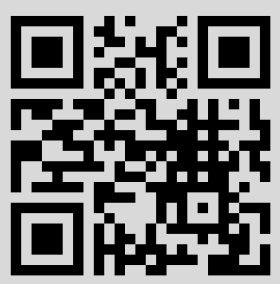


С другой стороны, если считать условие с членами ряда Кемпбелла-Хаусдорфа выполненным при всех $k$, то доказанная теорема даст некоторое обобщение одной из «абсолютных» теорем работы [4].

\title{
ЛитерАТУРА
}

1. Рудин У. Функциональный анализ. Мир, М., 1975. 2. Moore R. Proc. Amer. Math. Soc., 50, 138-155 (1975). 3. Горин E. A., Караханян М. И. Матем. заметки, 19, №2, 179-188 (1977). 4. Горин E. А. Алгебра и анализ, 5, вып. 4, 83-97 (1993). 5. Караханян М. И. Изв. АН Арм. ССР, матем., 19, №5, 405-421 (1978). 6. Караханян М. И. Изв. НАН Арм., матем., 29, № 1, 50-58 (1994).

Ереванский государственный университет e-mail: karakhan@ysu.am

Поступило в редакцию 12 апреля 2005 г.

УДК 517.9

\section{О некоторых функциональных уравнениях и представлениях топологических полугрупп}

\author{
(c) 2005. Е. В. ШульмАн
}

В данной работе некоторые результаты общего характера о представлениях топологических полугрупп применяются к исследованию следующего вопроса. Пусть заданы коммутативная топологическая полугруппа $G$ и натуральное число $n>1$. Требуется описать все непрерывные функции $f: G \rightarrow \mathbb{C}$, такие, что функция $\left(x_{1}, \ldots, x_{n}\right) \mapsto f\left(x_{1}+\cdots+x_{n}\right)$ полиномиально выражается через функции от сумм меньшего числа переменных. Говоря точнее, речь идет о следующем соотношении:

$$
f\left(x_{1}+\cdots+x_{n}\right)=\sum_{\gamma} \prod_{\Delta_{i} \in \gamma} a_{\Delta_{i}}^{\gamma}\left(\sum_{k \in \Delta_{i}} x_{k}\right)+\sum_{l=1}^{m} \alpha_{l}(x) \beta_{l}(y) \eta_{l}(z) .
$$

Здесь $\alpha_{l}, \beta_{l}, \eta_{l}(1 \leqslant l \leqslant m)$ - непрерывные функции на $G, \gamma$ пробегает всевозможные разбиения множества $\{1, \ldots, n\}=\Delta_{1} \cup \cdots \cup \Delta_{r}, r>1, \Delta_{i} \neq \varnothing$, а $a_{\Delta_{i}}^{\gamma}$ - непрерывные функции на $G$, «нумерованные» элементами $\Delta_{i}$ разбиения $\gamma$ (в каждом одночлене из правой части уравнения (1) участвуют лишь попарно не пересекающиеся наборы переменных). Таким образом, речь идет о функциях $f$, допускающих теорему сложения специального вида.

Частные случаи этой задачи изучались во многих работах (см., например, $[5,4]$ и цитированную там литературу). Наиболее важным из этих частных случаев являются уравнения Леви-Чивиты [1]

$$
f(x+y)=\sum_{i=1}^{n} a_{i}(x) b_{i}(y)
$$

В [1] было доказано, что при $G=\mathbb{R}$ решения этих уравнений - квазимногочлены. В общем случае эти решения можно описать как матричные элементы конечномерных представлений полугруппы $G$ (см. $[2,3])$, причем представления можно выбрать с сохранением таких свойств решений, как ограниченность или непрерывность. Мы распространим эти результаты на уравнение (1). 
Начнем с рассмотрения более общей ситуации. Пусть $G$ - топологическая полугруппа. Подмножество $E \subset G$ называется обратимо стабильным (IS-noдмножеством), если оно содержит всякий элемент $g \in G$, такой, что $g E \cap E \neq \varnothing$.

Легко проверить, что пересечение двух IS-подмножеств также является IS-подмножеством; поэтому для любого множества $E \subset G$ существует наименьшая замкнутая обратимо стабильная подполугруппа $G_{I S}(E)$, содержащая $E$. Если $G_{I S}(E)=G$, то мы говорим, что $E$ топологически слабо порождает $G$. Если к тому же $E$ конечно, то будем говорить, что $G$ mопологически слабо конечно порождена. Нетрудно видеть, что полугруппы $\mathbb{N}, \mathbb{R}^{n}, \mathbb{C}^{n}, \mathbb{R}_{+}^{n}$ являются топологически слабо конечно порожденными. Следует отметить, что если $G-$ группа, то слово «слабо» здесь излишне: $G$ является топологически слабо конечно порожденной тогда и только тогда, когда она содержит плотную конечно порожденную подгруппу.

Допустим, что задано представление $T$ полугруппы $G$ в линейном пространстве $X$. Вектор $x \in X$ назовем конечным, если его орбита $T(G) x$ содержится в конечномерном подпространстве.

Теорема 1. Пусть $G$ - топологически слабо конечно порожденная полугруппа и $T: G \rightarrow \mathscr{L}(X)$ - ее непрерьвное представление на топологическом векторном пространстве $X$. Пусть $x$ - произвольный вектор из $X u$ $\alpha(g)$ - непрерывная функиия на $G$, не принимающая нулевых значений. Если для каждого элемента $g \in G$ вектор $T_{g} x-\alpha(g) x$ конечен, то и $x$ конечен.

Теорема 2. Пусть непреръвное представление $T: G \rightarrow \mathscr{L}(X)$ топологически слабо конечно порожденной полугруппы $G$ таково, что для каждого характера $\lambda$ этой полугруппы соответствующее собственное подпространство $\left\{x \in X: T_{g} x=\lambda(g) x\right\}$ конечномерно. Тогда для любого конечномерного подпространства $L \subset X$ и любой не принимающей нулевых значений функиии $\alpha(g)$ на $G$ подпространство $\mathscr{R}(\alpha, L)=\left\{x \in X: T_{g} x-\alpha(g) x \in L\right.$ для любого $\left.g \in G\right\}$ конечномерно.

ЗАмЕчАниЕ 3. В теоремах 1 и 2 условие $\alpha(g) \neq 0$ можно опустить, если ни один нетривиальный характер полугруппы $G$ не принимает нулевых значений (как, например, в случае, когда $G$ - группа).

Следующий пример показывает, что условие слабой конечной порожденности в теореме 1 нельзя опустить, даже если ограничиться классом коммутативных групп.

Пусть $G$ - прямая сумма счетного числа экземпляров прямой $\mathbb{R}$, т. е. группа всех последовательностей вещественных чисел с конечным числом ненулевых элементов, наделенная топологией индуктивного предела (или же топологией поточечной сходимости). Пусть $T$ - представление группы $G$ на пространстве всех последовательностей (с топологией поточечной сходимости), определенное формулой

$$
T_{\left(a_{1}, a_{2}, \ldots\right)}\left(x_{1}, x_{2}, \ldots\right)=\left(\exp \left(a_{1}\right) x_{1}, \exp \left(a_{2}\right) x_{2}, \ldots\right) .
$$

Тогда легко видеть, что вектор $x=(1,1, \ldots)$ не является конечным, хотя все векторы $T_{a} x-x$ конечны.

Приведем также пример, показывающий, что и в теореме 2 условие слабой конечной порожденности существенно. Пусть $X$ - бесконечномерное банахово пространство, $L$ - его конечномерное подпространство и $G$ - группа всех 
непрерывных операторов вида $1+S$, где $S X \subset L, S L=0$. Тогда для тождественного представления и функции $\alpha$, тождественно равной 1 , подпространство $\mathscr{R}(\alpha, L)$ совпадает с $X$. Нетрудно видеть, что $\alpha$ - единственный характер группы $G$ и что соответствующее собственное подпространство совпадает с $L$.

Тем не менее некоторые полезные (при работе с функциональными уравнениями) модификации теоремы 1 справедливы и для полугрупп, не являющихся топологически слабо конечно порожденными. Отметим один простой результат, не использующий каких бы то ни было топологических условий.

Теорема 4. Пусть $T: G \rightarrow \mathscr{L}(X)$ - представление полугруппь $G$ в линейном пространстве $X$, такое, что для некоторого $h \in G$ число 1 не является собственным числом оператора $T_{h}$. Если вектор $T_{h} x-x$ конечен, то и $x$ конечен.

Заметим, что при решении функциональных уравнений в классе непрерывных функций этот результат неприменим, поскольку неподвижные точки там всегда есть. Однако при нахождении суммируемых или убывающих на бесконечности решений он оказывается полезным.

Будем обозначать через $\mathscr{M}(G)$ множество всех матричных элементов всех непрерывных конечномерных представлений полугруппы $G$. Нетрудно видеть, что каждая функция из $\mathscr{M}(G)$ удовлетворяет уравнению (1) при некотором $n$ (при этом все $a_{i}$ и $b_{i}$ можно взять равными нулю). Применяя теорему $1 \mathrm{k}$ регулярному представлению полугруппы $G$ в $C(G)$, можно показать, что этот класс исчерпывает все непрерывные решения уравнения (1):

ТеОрема 5. Пусть $G$ - коммутативная унитальная топологически слабо конечно порожденная полугруппа, и пусть непрерьвная функиия $f: G \rightarrow \mathbb{C}$ удовлетворяет уравнению (1). Тогда $f \in \mathscr{M}(G)$.

При малых $n$ к уравнению (1) сводятся некоторые уравнения более общего вида. Мы приведем лишь один из результатов такого рода:

СлЕДСТВИЕ 6. Пусть $G$ - коммутативная униталъная топологически слабо конечно порожденная полугруппа. Если непрерывная комплекснозначная функиия $f$ на $G$ удовлетворяет уравнению

$$
f(x+y+z)=a_{1}(x) b_{1}(y, z)+a_{2}(y) b_{2}(x, z)+a_{3}(z) b_{3}(x, y)+\sum_{k=1}^{m} \alpha_{k}(x) \beta_{k}(y) \gamma_{k}(z),
$$

mo $f \in \mathscr{M}(G)$.

Автор выражает искреннюю признательность рецензенту за полезные замечания и, в частности, за предложение записывать уравнение (1) в настоящей, более общей по сравнению с первоначальной, форме.

\section{ЛИтерАТУРА}

1. Levi-Cività T. Atti Accad. Naz. Lincei Rend. (5), 22, 181-183 (1913). 2. Shulman E. J. London Math. Soc., II Ser., 54, No. 1, 111-120 (1996). 3. Szekelehidi L. On the LeviChivita functional equation, Ber. Math.-Stat. Sekt. Forschungsges. Joanneum Graz 301, 1988. 4. Trif T. J. Math. Anal. Appl., 272, 604-616 (2002). 5. Chang Ick-Soon, Lee Eun Hwi, Kim Hark-Mahn. Math. Inequal. Appl., 6, No. 1, 87-95 (2003).

School of Mathematical Sciences,

Поступило в редакцию Tel-Aviv University, Israel 21 января 2004 г.

email: shulmank@yahoo.com 